\title{
Guideline on the use of onabotulinumtoxinA in chronic migraine: a consensus statement from the European Headache Federation
}

Lars Bendtsen ${ }^{1^{*}}$ (D), Simona Sacco ${ }^{2}$, Messoud Ashina ${ }^{1}$, Dimos Mitsikostas ${ }^{3}$, Fayyaz Ahmed ${ }^{4}$, Patricia Pozo-Rosich ${ }^{5,6}$ and Paolo Martelletti ${ }^{7}$

\begin{abstract}
OnabotulinumtoxinA is being increasingly used in the management of chronic migraine (CM). Treatment with onabotulinumtoxinA poses challenges compared with traditional therapy with orally administered preventatives. The European Headache Federation identified an expert group that was asked to develop the present guideline to provide recommendations for the use of onabotulinumtoxinA in CM. The expert group recommend onabotulinumtoxinA as an effective and well-tolerated treatment of CM. Patients should preferably have tried two to three other migraine prophylactics before start of onabotulinumtoxinA. Patients with medication overuse should be withdrawn from the overused medication before initiation of onabotulinumtoxinA if feasible, if not onabotulinumtoxinA can be initiated from the start or before withdrawal. OnabotulinumtoxinA should be administered according to the PREEMPT injection protocol, i.e. injecting $155 \mathrm{U}-195 \mathrm{U}$ to 31-39 sites every 12-weeks. We recommend that patients are defined as non-responders, if they have less than $30 \%$ reduction in headache days per month during treatment with onabotulinumtoxinA. However other factors such as headache intensity, disability and patient preferences should also be considered when evaluating response. Treatment should be stopped, if the patient does not respond to the first two to three treatment cycles. Response to continued treatment with onabotulinumtoxinA should be evaluated by comparing the 4 weeks before with the 4 weeks after each treatment cycle. It is recommended that treatment is stopped in patients with a reduction to less than 10 headache days per month for 3 months and that patients are re-evaluated 4-5 months after stopping onabotulinumtoxinA to make sure that the patient has not returned to CM. Questions regarding efficacy and tolerability of onabotulinumtoxinA could be answered on the basis of scientific evidence. The other recommendations were mainly based on expert opinion. Future research on the treatment of $\mathrm{CM}$ with onabotulinumtoxinA may further improve the management of this highly disabling disorder.
\end{abstract}

Keywords: Chronic migraine, OnabotulinumtoxinA, Management, Guideline

\footnotetext{
* Correspondence: lars.bendtsen@regionh.dk

1Danish Headache Center, Department of Neurology, Rigshospitalet-Glostrup,

Faculty of Health and Medical Sciences, University of Copenhagen, 2600

Glostrup, Denmark

Full list of author information is available at the end of the article
}

(c) The Author(s). 2018 Open Access This article is distributed under the terms of the Creative Commons Attribution 4.0 International License (http://creativecommons.org/licenses/by/4.0/), which permits unrestricted use, distribution, and reproduction in any medium, provided you give appropriate credit to the original author(s) and the source, provide a link to the Creative Commons license, and indicate if changes were made. 


\section{Introduction}

Chronic migraine (CM) is a debilitating disorder affecting approximately $2 \%$ of the general population [1] and is very common in specialised headache centres. CM is defined as headache occurring on $\geq 15$ days per month for $>$ 3 months of which $\geq 8$ days has the features of migraine headache [2]. Only two treatments have demonstrated efficacy in CM: onabotulinumtoxinA and topiramate [3].

Possible efficacy of onabotulinumtoxinA in migraine was incidentally noted in patients treated cosmetically for wrinkles. In 2010, onabotulinumtoxinA was reported effective for the treatment of CM in the Phase 3 Research Evaluating Migraine Prophylaxis Therapy (PREEMPT) trials [4,5] and was approved both by the European Medicines Agency and by the US Food and Drug Administration for the prophylaxis of CM. Its use was endorsed by the National Institute for Health and Care Excellence (NICE) in 2012 [6]. OnabotulinumtoxinA has not been found effective in episodic migraine or in tension-type headache [7]. The mode of action of onabotulinumtoxinA in CM may include modulation of neurotransmitter release, changes in surface expression of receptors and cytokines as well as enhancement of opioidergic transmission [8]. It is likely that onabotulinumtoxinA reduces both peripheral and central sensitization in CM through such mechanisms [7, 9].

Since its approval, the use of onabotulinumtoxinA has increased considerably. Treatment with onabotulinumtoxinA poses challenges compared with traditional therapy with orally administered preventatives for headache specialists not used to injecting toxins. Due to the absence of European Guidelines for the use of onabotulinumtoxinA, the European Headache Federation has considered the need to write a Guideline for the use of onabotulinumtoxinA in CM.

The aim of this guideline is to provide recommendations for the use of onabotulinumtoxinA in CM.

\section{Methods}

The EHF identified an expert panel consisting of seven members. We have developed recommendations for a series of questions that are essential for daily clinical use of onabotulinumtoxin A in CM, which are based on the available evidence and our clinical experience.

We have answered the following questions:

1. Is onabotulinumtoxinA effective and well-tolerated for the treatment of CM?

2. When should onabotulinumtoxinA be offered?

3. Should withdrawal be performed before treatment with onabotulinumtoxinA in patients with $\mathrm{CM}$ and medication-overuse?

4. How should the treatment be administered?

5. When can a de novo patient be considered nonresponder to onabotulinumtoxinA?
6. How should responders to onabotulinumtoxinA be managed over time?

In the discussion section we have summarized our findings and added some general considerations.

The Grading of Recommendations, Assessment, Development and Evaluation (GRADE) system has been endorsed by the European Academy of Neurology [10] as the method of choice to establish recommendations and was used here if possible as was the Patients; Intervention; Comparison and Outcome (PICO) [10] method. Final quality of evidence was rated as high, moderate, low or very low based on study design, study limitations, inconsistency, indirectness, imprecision, publication bias, effect size, dose response and confounding. Strength (strong or weak) and direction (for or against) of recommendation were determined on basis of balance between desirable and undesirable effects, quality of evidence, values and preferences and costs [10]. With our present scientific evidence this could only be used for the first question. If GRADE was not applicable, an ungraded good practice statement was given, according to the available level of evidence. The Delphi method was used to reach consensus.

\section{Search strategy and results}

Papers published in peer-reviewed journals were identified using PubMed/Medline, EMBASE and Cochrane Library. Search was done with unrestricted date of start and until April 2018 and restricted to English language. We searched for prospective studies investigating efficacy, safety or tolerability of onabotulinumtoxinA in CM compared with placebo or other prophylactic treatments and for prospective observational studies published in peer-reviewed journals. We excluded retrospective studies, studies not performed according to PREEMPT, studies of poor quality, e.g. studies with insufficient reporting of diagnostic criteria or outcome and reviews.

We initially identified 823 studies which were finally reduced to 27 studies. Please see Fig. 1. The retrieved studies were all considered for question 1. For the following questions search was performed among the retrieved studies.

\section{Recommendations}

Question 1: For patients with CM is treatment with onabotulinumtoxinA effective and well-tolerated? PICO:

Population: patients with CM with or without medication overuse Intervention: onabotulinumtoxinA $155 \mathrm{U}$ to $195 \mathrm{U}$ Comparison: placebo or other prophylactic treatments 


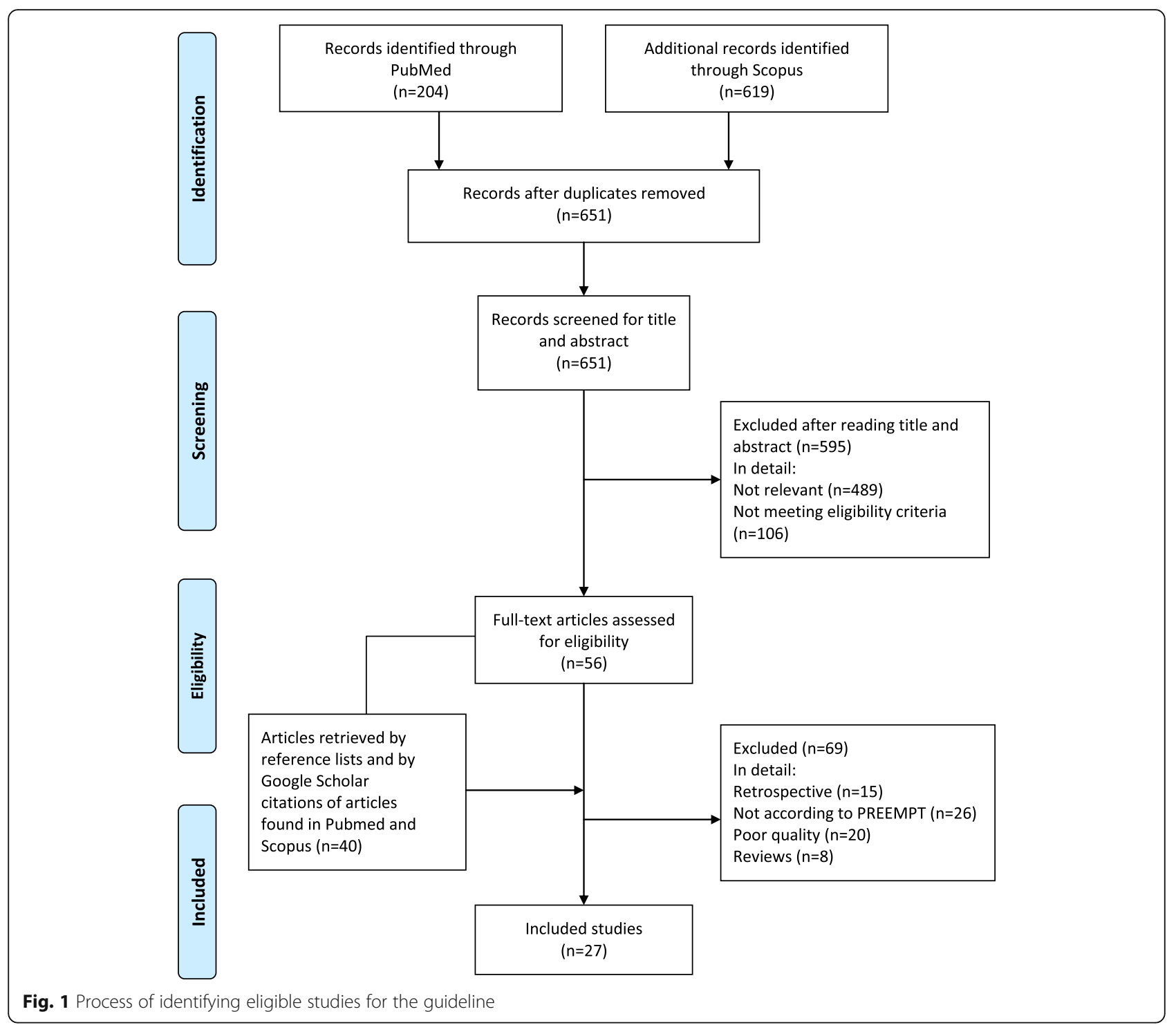

Outcome: meaningful reduction of headache days ( $\geq 30 \%$ from baseline) with acceptable side-effects

\section{Search strategy and results}

We identified two randomized placebo-controlled multicentre trials, the PREEMPT 1 [4] and PREEMPT 2 [5] (Phase 3 Research Evaluating Migraine Prophylaxis Therapy) clinical trials. Both trials consisted of a 24-week placebo-controlled phase followed by a 32-week open-label extension phase. OnabotulinumtoxinA was administered at 12-week intervals. OnabotulinumtoxinA was injected in 31 sites (5 $\mathrm{U}$ per injection) with possibility for additional 8 injections according to a "follow-the pain" strategy. The total dose of onabotulinumtoxinA was $155 \mathrm{U}$ to $195 \mathrm{U}$ injected in 31 to 39 sites. This is hereafter referred to as the PREEMPT injection protocol. A total of 1384 patients received onabotulinumtoxinA or placebo. Approximately two-thirds of $\mathrm{CM}$ patients were overusing headache medications at baseline. There was no significant effect on the primary endpoint, headache episodes, in the PREEMPT 1 trial, while there was a significant effect on headache and migraine days. The PREEMPT 2 trial met its primary endpoint headache days. The results of the PREEMPT 1 and 2 trials have been evaluated in a pooled analysis [11]. The pooled analysis reported a large decrease from baseline in headache days, but due to a large placebo-effect, the efficacy of onabotulinumtoxinA over placebo was modest (8.4 vs. -6.6 days per 4 weeks, $P<0.01$ ) [11]. Responder rate, defined as percentage of patients with a decrease in frequency of headache days from baseline of at least $50 \%$, was $47.1 \%$ vs. $35.1 \%(P<0.001)$. Post-hoc analysis demonstrated that $71.4,9.4$ and $5.4 \%$ of patients responded to treatment cycles 1,2 and 3 respectively using 30\% reduction of headache days compared with baseline as responder rate [12]. 
OnabotulinumtoxinA was also significantly more effective in reducing a number of secondary efficacy variables including disability. The Headache Impact Test (HIT)-6 score was reduced by 4.8 by onabotulinumtoxinA compared with 2.4 by placebo $(P<0.001)$. Post-hoc analyses demonstrated that headache intensity was reduced in non-responders (patients with less than $50 \%$ reduction in headache frequency) [13] and that quality of life was still improved after 1 year of treatment [14]. OnabotulinumtoxinA was well tolerated, the most common adverse events compared with placebo were neck pain $(6.7 \%$ vs. $2.2 \%)$, muscular weakness $(5.5 \%$ vs. $0.3 \%)$, eyelid ptosis ( $3.3 \%$ vs. $0.3 \%)$ and injection-site pain $(3.2 \%$ vs. $2.0 \%)$. Discontinuation rates due to adverse events were low (3.8\% vs. $1.2 \%)$. A secondary analysis of the PREEMPT study investigating the subgroup of patients (1005 out of 1384) who received all 5 treatment cycles demonstrated continued efficacy and tolerability of onabotulinumtoxinA [15].

A subgroup analysis of 904 patients with medication overuse from the PREEMPT studies found similar effect in patients with and without medication overuse [16]. When evaluating these data it should be noted that they were obtained from post hoc analysis [17]. The same was found in a UK study comparing efficacy of onabotulinumtoxinA administered according to PREEMPT in 219 patients with and 215 patients without medication overuse [18].

The good safety and tolerability profile of onabotulinumtoxinA was confirmed in a pooled analysis [19] of two phase 2 studies in chronic daily headache $[20,21]$ and the two PREEMPT studies [4,5]. Furthermore, a sub-analysis of 513 subjects receiving all 5 treatment cycles in the PREEMPT studies found that adverse events decreased over time [19].

We identified a number of prospective open-label studies evaluating efficacy and safety of onabotulinumtoxinA [22-39]. In general these studies were long-term studies treating CM patients with and without medication overuse according to the PREEMPT protocol. Several of the studies are limited by some methodological flaws [40]. These studies consistently reported positive results regarding both efficacy and tolerability in line with the PREEMPT studies. A UK study including 254 patients reported responder rates of $32 \%$ and $47 \%$ when response was defined as $\geq 50 \%$ and $\geq 30 \%$ reduction in headache days, respectively [30]. A large multinational study including 1160 patients confirmed the positive safety profile of onabotulinumtoxinA [41]. Longest follow-up reported was in the multinational Chronic migraine OnabotulinuMtoxinA Prolonged Efficacy open Label (COMPEL) study [23], which included 716 patients of whom 373 completed the 2 years follow-up.

A randomized controlled study reported better efficacy of acupuncture than of onabotulinumtoxin A and lowdose valproate [33]. Two studies reported comparable efficacy of onabotulinumtoxinA and topiramate [42, 43] with fewer adverse events from onabotulinumtoxinA [43]. The latter is supported by clinical experience.

Further evidence is under way through the REsource utilisation and Patient-reported OutcomeS (REPOSE) studies [44].

\section{Clinical guide}

The blinding of the PREEMPT studies has been criticized, because injections in the forehead removing wrinkling could cause un-blinding $[6,7]$. In an older study $70 \%$ and $60 \%$ of patients who knew that they had a $50 \%$ chance of receiving placebo, correctly guessed that they were treated with active drug after the first and third treatment cycles respectively [20]. According to GRADE (Table 1) the quality of the PREEMPT studies regarding efficacy is moderate due to risk of un-blinding, while it is high regarding safety because of the very positive safety profile. The high placebo response in the PREEMPT studies [11] and the long-term effect of onabotulinumtoxinA [23] speak against placebo being a major factor.

Prospective open-label observational studies have serious limitations regarding evaluation of efficacy including lack of control group, risk of publication bias and a potentially high placebo-response. However, the results from the studies are quite consistently positive and support the findings from the placebo-controlled PREEMPT studies. In addition they provide important information on efficacy and tolerability of long-term treatment with onabotulinumtoxinA.

Table 1 GRADE evaluation of placebo-controlled studies evaluating efficacy and tolerability of onabotA

\begin{tabular}{|c|c|c|c|c|c|c|c|c|c|}
\hline $\begin{array}{l}\text { Studies } \\
\text { (participants) }\end{array}$ & Outcome & Comparison & Type & Quality & $\begin{array}{l}\text { Effect } \\
\text { size }\end{array}$ & $\begin{array}{l}\text { GRADE quality } \\
\text { of evidence }\end{array}$ & Direction & Strength & Comment \\
\hline Dodick (1384) & Headache days & $\begin{array}{l}\text { OnabotA } 155 \mathrm{U}-195 \mathrm{U} \\
\text { versus placebo }\end{array}$ & $R$ & -1 & 0 & Moderate & For & Strong & $\begin{array}{l}\text { Quality points deducted for risk } \\
\text { of bias }(-1)\end{array}$ \\
\hline Diener (2436) & Adverse events & $\begin{array}{l}\text { OnabotA } 75 \mathrm{U}-260 \mathrm{U} \\
\text { versus placebo }\end{array}$ & $\mathrm{R}$ & -1 & +1 & High & For & Strong & $\begin{array}{l}\text { Quality points deducted for risk } \\
\text { of bias }(-1) \text {. Effect size point } \\
\text { added for small frequency of } A E\end{array}$ \\
\hline
\end{tabular}

The Dodick paper [11] was a pooled analysis of the Aurora [4] and 2010 Diener [5] studies. The 2014 Diener paper [19] was a pooled analysis of two phase 2 studies in chronic daily headache $[20,21]$ and the two PREEMPT studies $[4,5]$. Type: $R$ randomized controlled trial 
Taking the collected evidence from the PREEMPT studies, the observational studies and clinical experience into consideration, the final quality of evidence is considered to be high regarding both efficacy and safety of onabotulinumtoxinA. The effect size in average is considered modest compared with placebo. However, average group differences in efficacy cannot be considered in isolation as this may obscure meaningful individual patient differences. Direction of recommendation for use of onabotulinumtoxinA is for and strength of recommendation is strong. Quality of evidence for comparison of onabotulinumtoxinA with other prophylactic treatments is too low to make final conclusions, but it is possible that the effect size is comparable to that of topiramate but with a better tolerability profile.

\section{Final recommendation}

OnabotulinumtoxinA is recommended for treatment of patients with $\mathrm{CM}$ and considered an effective and welltolerated treatment. Quality of evidence: high. Strength of the recommendation: strong.

\section{Clinical question 2: For patients with $\mathrm{CM}$ when should onabotulinumtoxinA be offered? \\ Search strategy and results}

Among the retrieved studies, we searched for reports evaluating how onabotulinumtoxinA candidates should be selected. We identified the following relevant questions:

How many prophylactics should have failed before onabotulinumtoxinA is administered? NICE performed a subgroup analysis of efficacy related to previous treatment with prophylactic medications. They found that onabotulinumtoxinA was equally effective in patients who had previously received one, two or three or more preventive treatments [6]. Based on cost-effectiveness in the UK, NICE recommends that patients should have failed three or more other migraine prophylactics before onabotulinumtoxinA is administered [6].

Is it possible to predict who will be good responders? It has been reported that short disease duration [28, 32] and high serum levels of calcitonin gene-related peptide (CGRP) [27] are predictors of good outcome to onabotulinumtoxinA.

\section{Clinical guide}

Taking costs into consideration, failure of at least three migraine prophylactics before onabotulinumtoxinA is administered is recommended by NICE. However, this may not apply to all countries, and some experts recommend initiation of onabotulinumtoxinA after failure of at least two to three prophylactics, because topiramate is the only other drug with proved efficacy in CM and because of the better tolerability profile of onabotulinumtoxinA. We recommend that patients should have failed (lack of effect or intolerable side-effects) two to three or more other migraine prophylactics before onabotulinumtoxinA is administered. However, in some patients this is not possible due to multiple comorbid disorders, e.g., cardiac disorder, overweight or depression. Some data indicate that patients who are treated earlier have a better response. However, early stage CM may be more likely to undergo spontaneous fluctuations and/or to improve spontaneously than later stage CM. Yet, there are not enough data to select CM patients to be or not to be treated with onabotulinumtoxinA based on clinical or laboratory characteristics.

\section{Final recommendation}

It is recommended that patients should have failed at least two to three other migraine prophylactics unless contraindicated by comorbid disorders before onabotulinumtoxinA is administered. This recommendation is based on expert opinion.

Question 3: For patients with CM plus medication-overuse should withdrawal be done before treatment with onabotulinumtoxinA is initiated?

\section{Search strategy and results}

Among the retrieved studies we searched for reports comparing treatment with onabotulinumtoxinA initiated before as compared with after withdrawal of the overused medication in patients with $\mathrm{CM}$ plus medication overuse. We found no such studies.

\section{Clinical guide}

There is general consensus that CM patients with medication overuse should be withdrawn from the overused medication (detoxified) [6, 17] but not how this should be done. Evidence indicate that detoxification both with onabotulinumtoxinA [16, 18], detoxification with or without oral prophylactics from the start [45], and detoxification with delayed prophylactic treatment after 2 months [46] are all effective. Because of differences in, e.g., study design and patients characteristics, it is not possible to compare efficacy among these studies. Considering that not all patients need prophylactics after detoxification [47], it is recommended to detoxify first with later initiation of onabotulinumtoxinA when possible. This is in accordance with recommendations from (NICE) [6]. If this is not possible, onabotulinumtoxinA can be initiated from the start or even before withdrawal of the overused medication.

\section{Final recommendation}

In patients with CM and medication overuse, it is preferable to detoxify first with later initiation of onabotulinumtoxinA. If this is not feasible, onabotulinumtoxinA can be initiated from the start or even before withdrawal 
of the overused medication. This recommendation is based on expert opinion.

\section{Clinical question 4: How should treatment with onabotulinumtoxinA be administered? Search strategy and results}

Among the retrieved studies, we searched for reports evaluating administration of onabotulinumtoxinA.

The PREEMPT injection protocol, i.e. injection of $155 \mathrm{U}-$ $195 \mathrm{U}$ administered to 31-39 sites every 12-weeks as previously described, set the standard for treatment with onabotulinumtoxin A in CM. It has been argued that an injection paradigm customized to the individual patient would be preferable [48]. However, we found no studies comparing the PREEMPT protocol with an alternative protocol.

Treatment with $155 \mathrm{U}$ and $195 \mathrm{U}$ has not been compared head to head. In an open label prospective study, Negro et al. [35] reported higher effect of $195 \mathrm{U}$ than of $155 \mathrm{U}$ given in an earlier study from the same study group.

\section{Clinical guide}

The PREEMPT injection protocol should be followed, since it is the only protocol that has proved efficacy of onabotulinumtoxinA. It is possible that $195 \mathrm{U}$ is more effective than $155 \mathrm{U}$. The higher dose could be considered, if the patient does not respond to $155 \mathrm{U}$.

\section{Final recommendation}

OnabotulinumtoxinA should be administered according to the PREEMPT injection protocol. This recommendation is based on evidence from the PREEMPT trials.

\section{Clinical question 5: When can a de novo patient be considered non-responder to onabotulinumtoxinA? Search strategy and results}

Among the retrieved studies, we searched for reports evaluating when patients previously naïve to onabotulinumtoxinA can be considered non-responders.

In the PREEMPT studies responders were defined as patients with at least $50 \%$ reduction in headache days per month, while NICE defined responders as patients having at least $30 \%$ reduction in headache days per month [6]. Like in other chronic pain conditions [49], most headache experts regard a 30\% reduction in headache days for clinically relevant in a complicated disorder as CM [12, 50]. Khalil et al. [30] suggested that responders should be defined as either $50 \%$ or more reduction in headache or migraine days or doubling of headache-free days ("crystal clear days") provided that there was at least 3 headache-free days at baseline. Other experts consider $\mathrm{a}<30 \%$ reduction in headache days as response, if it is accompanied by improvement of other variables such as reduction in headache intensity or improvement of quality of life [50].
A pooled sub-analysis of the PREEMPT studies reported that among onabotulinumtoxinA treated patients $49.3,11.3$ and $10.3 \%$ of the patients responded for the first time (50\% responders) during treatment cycles 1,2 and 3 respectively [12]. These data were not tested against placebo. The authors suggested that patients should be offered 2-3 treatment cycles before being categorised as non-responders. Prospective open-label studies reported increased efficacy over 5-9 treatment cycles [35, 38]. NICE recommends that treatment should be stopped if the patient does not respond with at least $30 \%$ reduction in headache days per month after two treatment cycles [6].

\section{Clinical guide}

Response to onabotulinumtoxinA should be continuously monitored by headache calendars. The definition of responders to onabotulinumtoxinA is important for daily clinical practice, but there are currently no convincing data supporting one definition over another. We recommend the use of the definition most easy to use, i.e. that patients should be defined as non-responders, if they have less than $30 \%$ reduction in headache days during the first month after treatment with onabotulinumtoxinA compared with the month before first treatment. However, other factors such as headache intensity, disability and patient preferences should also be considered. Treatment should be stopped if the patient does not respond to onabotulinumtoxinA during the first 2-3 treatment cycles (negative stopping rule).

\section{Final recommendation}

It is recommended that patients should be defined as non-responders, if they have less than $30 \%$ reduction in headache days per month during treatment with onabotulinumtoxinA. However, other factors such as headache intensity, disability and patient preferences should also be considered. Treatment should be stopped, if the patient does not respond to the first 2-3 treatment cycles. This recommendation is based on expert opinion.

\section{Clinical question 6: How should responders to onabotulinumtoxinA be managed over time? Search strategy and results}

Among the retrieved studies, we searched for reports investigating when treatment with onabotulinumtoxinA should be stopped once started and how the patients should be managed over time. We found no such studies.

\section{Clinical guide}

Response to onabotulinumtoxinA should be continuously monitored by headache calendars, where patients record headache days and intake of acute headache medications. Available evidence indicate that electronic calendars, 
accessible via mobile technologies, may ensure better data recording [45].

There is no generally accepted agreement on from which time point's response to onabotulinumtoxinA should be evaluated during continued treatment. We suggest that the 4 weeks before and 4 weeks after each treatment cycle should be compared.

NICE recommends that treatment with onabotulinumtoxin A is stopped if the patient has reverted to episodic migraine for three consecutive months [6] (positive stopping rule). This may be impractical in many clinics, because it can be impossible to offer the patient a fast appointment if migraine reverts to $\mathrm{CM}$ due to lack of resources. The delay may result in worsening of headache and decreased quality of life [50]. The natural fluctuations occurring over time in CM [51] should also be taken into account. It has been proposed that onabotulinumtoxinA is stopped only in patients with a reduction to less than 10 headache days per month for 3 months given that those with a higher frequency carry a higher risk of relapse to $\mathrm{CM}$ [7]. Others have recommended that only in those subjects who are stable responders to onabotulinumtoxinA for at least 1 year, the extension of the inter-injection interval may be a responsible strategy to verify whether the improvement is a long-lasting remission of the disease or attenuation of symptoms due to onabotulinumtoxinA treatment [40].

We recommend that patients are re-evaluated 45 months after stopping onabotulinumtoxinA to make sure that the patient has not returned to CM.

\section{Final recommendations}

It is recommended to evaluate the response to continued treatment with onabotulinumtoxinA on the basis of headache calendars by comparing the 4 weeks before and 4 weeks after each treatment cycle. We recommend to stop treatment in patients with a reduction to less than 10 headache days per month for 3 months. However, other factors such as headache intensity, disability and patient preferences should also be considered. Patients should be re-evaluated 4-5 months after stopping onabotulinumtoxinA to make sure that the patient has not returned to $\mathrm{CM}$. These recommendations are based on expert opinion.

\section{Discussion}

OnabotulinumtoxinA is recommended as an effective and well-tolerated treatment of CM. Patients should preferably have tried 2-3 other migraine prophylactics before start of onabotulinumtoxinA. However, many CM patients bear a considerable load of co-morbidities [52]. This can make it challenging to choose oral prophylactics, since many of these are contraindicated in the presence of, e.g., cardiovascular disease, depression or obesity. Treatment with oral prophylactics is often further complicated by poor tolerability, resulting in low persistence to oral prophylactic treatment in migraineurs [53]. If the patient has some, but insufficient, effect of oral prophylactics, these can go hand in hand with onabotulinumtoxinA if needed.

Management of concomitant medication overuse is controversial and differs considerably among headache centres and countries. At present we have no robust data that favours one approach over another. We recommend that patients with medication overuse should be withdrawn from the overused medication before initiation of onabotulinumtoxinA if feasible, if not onabotulinumtoxinA can be initiated from the start or before withdrawal.

Before the PREEMPT studies, onabotulinumtoxinA was administered according to highly variable protocols in the published reports, e.g. with different doses, number of injection sites and intervals between treatment cycles, resulting in inconsistent results. We recommend that onabotulinumtoxinA is administered according to the PREEMPT injection protocol, i.e. injecting $155 \mathrm{U}-$ $195 \mathrm{U}$ to $31-39$ sites every 12-weeks, because this is the only treatment paradigm that has scientifically proved to be effective. It is possible that $195 \mathrm{U}$ is more effective than $155 \mathrm{U}$. The higher dose could be considered, if the patient does not respond to $155 \mathrm{U}$.

The success of treatment depends not only on how onabotulinumtoxin A is administered but also on how the patient is treated in other aspects. Patients should be educated about their condition and how onabotulinumtoxinA is administered. Patients should be given realistic expectations, i.e. they should be told that the treatment may be able to reduce frequency and intensity of their migraine, but that it does not cure migraine. They should be told that effect usually is seen within 3-7 days, that the effect may wear off before the next treatment cycle, and that response should be evaluated by continued use of headache calendars.

Evaluation of response to migraine treatment is complex, because it involves not only frequency but also severity of headaches, tolerability of the treatment, disability and patient preferences. All of these factors should be taken into consideration when evaluating the response to onabotulinumtoxinA. However, there are no robust data showing which of these variables that best quantifies response to onabotulinumtoxinA. Furthermore, it is impractical for the patients to have to record many variables over a prolonged period of time. We therefore recommend that the simplest measure, headache days, is used as a minimum to monitor response. Other measures such as HIT-6 can be added. We recommend that patients are defined as non-responders, if they have less than $30 \%$ reduction in headache days per month during treatment with onabotulinumtoxin $\mathrm{A}$, and 
that treatment should be stopped, if the patient does not respond to the first 2-3 treatment cycles.

For initial responders to onabotulinumtoxinA, continued evaluation of response is complicated by the fact that the effect of treatment wears off after some time, typically 2 3 months, so which time points should the evaluation be based on? To complicate it more, most responders will not fulfil the criteria for $\mathrm{CM}$ when successfully treated, because they will not have had $\mathrm{CM}$ for more than 3 months when they show up for the next treatment cycle. So when should treatment be stopped? There are no robust data to guide us on these questions. We recommend that response to continued treatment with onabotulinumtoxin $\mathrm{A}$ is evaluated by comparing the 4 weeks before and 4 weeks after each treatment cycle. It is recommended that treatment is stopped in patients with a reduction to less than 10 headache days per month for 3 months and that patients are re-evaluated 45 months after stopping onabotulinumtoxinA to make sure that the patient has not returned to CM.

Cost-effectiveness of treatment with onabotulinumtoxinA is an important issue both for the individual and for society. NICE have calculated that treatment with onabotulinumtoxin A is cost-effective, if certain inclusion criteria and stopping rules are adhered to [6]. Likewise, reports from Italy [54], the US [55] and UK [56, 57] claim onabotulinumtoxinA to be cost effective. However, due to the highly variable health care systems in the European countries, it is not possible for us to give general evaluations on the cost-effectiveness of onabotulinumtoxinA.

\section{Conclusions and recommendations for future research}

The experts in the present panel are confident that onabotulinumtoxinA has an important role in the management of CM provided that the recommendations in this guideline are followed. However, only the first of the clinical questions in this guideline, regarding efficacy and tolerability of onabotulinumtoxinA, could be answered on the basis of scientific evidence.

There is a need for studies investigating the role of onabotulinumtoxinA in relation to other prophylactics, including the future calcitonin-gene-related-peptide receptor antagonists, and in relation to withdrawal of medication overuse. Furthermore there are important issues to be better analysed in relation to management with onabotulinumtoxinA over time, including optimal definition of de novo non-responders to onabotulinumtoxinA and even more challenging to decide when and how onabotulinumtoxinA should be considered to be tapered off in the long term management of initial responders.

Future research regarding the treatment of $\mathrm{CM}$ with onabotulinumtoxinA may further improve the management of this highly disabling disorder.

\begin{abstract}
Abbreviations
CM: Chronic migraine; GRADE: Grading of Recommendations, Assessment, Development and Evaluation; NICE: National Institute for Health and Care Excellence; PICO: Patients; Intervention; Comparison and Outcome;

PREEMPT: Phase 3 Research Evaluating Migraine Prophylaxis Therapy
\end{abstract}

\begin{abstract}
Acknowledgements
This article, as a Consensus Article from experts in the topic, has been reviewed internally among Authors. Its importance in the field and its suitability to be published in The Journal of Headache and Pain has been evaluated and confirmed by an independent The Journal of Headache and Pain Editorial Board Member.
\end{abstract}

\section{Funding}

The literature search was funded by a grant from the European Headache Federation.

\section{Availability of data and materials \\ Not applicable.}

\section{Authors contributions}

LB drafted the manuscript. All co-authors commented the manuscript. All authors read and approved the final manuscript.

Ethics approval and consent to participate Not applicable.

\section{Consent for publication}

Not applicable.

\section{Competing interests}

LB: Scientific advisory board for Novartis, Allergan and Biogen, principal investigator for Biogen and lectures for Allergan, Novartis and Teva. SS: Scientific advisory board for Allergan, Bayer Healthcare, Medtronic. MA: Personal fees from Alder BioPharmaceuticals, Allergan, Amgen, Alder, Eli Lilly, Novartis and Teva. Participated in clinical trials as the principal investigator for Alder ALD403-CLIN-011 (Phase 3b), Amgen 20,120,178 (Phase 2), 20,120,295 (Phase 2), 20,130,255 (OLE), 20,120,297 (Phase 3), GM-11 gamma-Core-R trials, Novartis CAMG334a2301 (Phase 3b), Amgen PAC1 20,150,308 (Phase 2a), Teva TV48125-CNS-30068 (Phase 3). Serves as associated editor of Cephalalgia, co-editor of the Journal of Headache and Pain.

DM: Scientific advisory board for Novartis, Eli Lilly, Sanofi-Genzyme and Teva. Principal investigator for Biogen, Cephaly, Genesis Pharma, Merck-Serono, Merz, Novartis and Teva. Has received honoraria for lecturing from Allergan, Amgen, Cephaly, Biogen, Genesis Pharma, Novartis, Rosche, Sanofi-Genzyme, Teva. FA: Consultant for Novartis, Allergan, Eneura and Electrocore (honorarium paid to the British Association for the Study of Headache and the Migraine Trust). PPR: Consultant and speaker for Allergan, Almirall, Chiesi, Eli Lilly, Novartis and Teva. Her research group has received research grants from Allergan and Novartis and has received funding for clinical trials from Alder, Boeringher Ingelheim, MSD, Electrocore, Eli Lilly, Janssen Cilag, Novartis and Teva. PM: Scientific Advisory Board for Allergan, Amgen, Electrocore, Eli Lilly, Novartis, Springer HealthCare, Teva.

\section{Publisher's Note}

Springer Nature remains neutral with regard to jurisdictional claims in published maps and institutional affiliations.

\section{Author details}

${ }^{1}$ Danish Headache Center, Department of Neurology, Rigshospitalet-Glostrup, Faculty of Health and Medical Sciences, University of Copenhagen, 2600 Glostrup, Denmark. ${ }^{2}$ Neuroscience Section, Department of Applied Clinical Sciences and Biotechnology, University of L'Aquila, 67100 L'Aquila, Italy. ${ }^{3} 1$ st Department of Neurology, Aeginition Hospital, National and Kapodistrian University of Athens, Athens, Greece. ${ }^{4}$ Department of Neurosciences, Hull York Medical School, Hull, UK. ${ }^{5}$ Headache \& Craniofacial Pain Unit, Neurology Department, Hospital Universitari Vall d'Hebron, Barcelona, Spain. ${ }^{6}$ Headache Research Group, Vall d'Hebron Research Institute (VHIR), Universitat Autònoma de Barcelona, Barcelona, Spain. ${ }^{7}$ Department of Clinical and Molecular Medicine, Sapienza University, Rome, Italy. 
Received: 22 July 2018 Accepted: 20 September 2018 Published online: 26 September 2018

\section{References}

1. Natoli JL, Manack A, Dean B et al (2010) Global prevalence of chronic migraine: a systematic review. Cephalalgia 30:599-609

2. Headache Classification Committee of the International Headache Society (IHS) (2018) The international classification of headache disorders, 3rd edition. Cephalalgia 38:1-211

3. Dodick DW, Turkel CC, DeGryse RE et al (2015) Assessing clinically meaningful treatment effects in controlled trials: chronic migraine as an example. J Pain 16:164-175

4. Aurora SK, Dodick DW, Turkel CC et al (2010) OnabotulinumtoxinA for treatment of chronic migraine: results from the double-blind, randomized, placebo-controlled phase of the PREEMPT 1 trial. Cephalalgia 30:793-803

5. Diener HC, Dodick DW, Aurora SK et al (2010) OnabotulinumtoxinA for treatment of chronic migraine: results from the double-blind, randomized, placebo-controlled phase of the PREEMPT 2 trial. Cephalalgia 30:804-814

6. NICE technology appraisal guidance 260 (2012) Botulinum toxin type A for the prevention of headaches in adults with chronic migraine. Available from https:// www.nice.org.uk/guidance/ta260

7. Gooriah R, Ahmed F (2015) OnabotulinumtoxinA for chronic migraine: a critical appraisal. Ther Clin Risk Manag 11:1003-1013

8. Do TP, Hvedstrup J, Schytz HW (2018) Botulinum toxin: a review of the mode of action in migraine. Acta Neurol Scand 137:442-451

9. Gazerani P, Pedersen NS, Staahl C, Drewes AM, Arendt-Nielsen L (2009) Subcutaneous botulinum toxin type a reduces capsaicin-induced trigeminal pain and vasomotor reactions in human skin. Pain 141:60-69

10. Leone MA, Brainin M, Boon P, Pugliatti M, Keindl M, Bassetti CL (2013) Guidance for the preparation of neurological management guidelines by EFNS scientific task forces - revised recommendations 2012. Eur J Neurol 20: 410-419

11. Dodick DW, Turkel CC, DeGryse RE et al (2010) OnabotulinumtoxinA for treatment of chronic migraine: pooled results from the double-blind, randomized, placebo-controlled phases of the PREEMPT clinical program. Headache 50:921-936

12. Silberstein SD, Dodick DW, Aurora SK et al (2015) Per cent of patients with chronic migraine who responded per onabotulinumtoxinA treatment cycle: PREEMPT. J Neurol Neurosurg Psychiatry 86:996-1001

13. Matharu M, Halker R, Pozo-Rosich P, Degryse R, Manack AA, Aurora SK (2017) The impact of onabotulinumtoxinA on severe headache days: PREEMPT 56-week pooled analysis. J Headache Pain 18:78

14. Lipton RB, Rosen NL, Ailani J, DeGryse RE, Gillard PJ, Varon SF (2016) OnabotulinumtoxinA improves quality of life and reduces impact of chronic migraine over one year of treatment: pooled results from the PREEMPT randomized clinical trial program. Cephalalgia 36:899-908

15. Aurora SK, Dodick DW, Diener HC et al (2014) OnabotulinumtoxinA for chronic migraine: efficacy, safety, and tolerability in patients who received all five treatment cycles in the PREEMPT clinical program. Acta Neurol Scand 129:61-70

16. Silberstein SD, Blumenfeld AM, Cady RK et al (2013) OnabotulinumtoxinA for treatment of chronic migraine: PREEMPT 24-week pooled subgroup analysis of patients who had acute headache medication overuse at baseline. J Neurol Sci 331:48-56

17. Chiang CC, Schwedt TJ, Wang SJ, Dodick DW (2016) Treatment of medication-overuse headache: a systematic review. Cephalalgia 36:371-386

18. Ahmed F, Zafar HW, Buture A, Khalil M (2015) Does analgesic overuse matter? Response to OnabotulinumtoxinA in patients with chronic migraine with or without medication overuse. Springerplus 4:589

19. Diener HC, Dodick DW, Turkel CC et al (2014) Pooled analysis of the safety and tolerability of onabotulinumtoxinA in the treatment of chronic migraine. Eur J Neurol 21:851-859

20. Mathew NT, Frishberg BM, Gawel M, Dimitrova R, Gibson J, Turkel C (2005) Botulinum toxin type a (BOTOX) for the prophylactic treatment of chronic daily headache: a randomized, double-blind, placebo-controlled trial. Headache 45:293-307

21. Silberstein SD, Stark SR, Lucas SM, Christie SN, DeGryse RE, Turkel CC (2005) Botulinum toxin type a for the prophylactic treatment of chronic daily headache: a randomized, double-blind, placebo-controlled trial. Mayo Clin Proc 80:1126-1137
22. Aydinlar El, Dikmen PY, Kosak S, Kocaman AS (2017) OnabotulinumtoxinA effectiveness on chronic migraine, negative emotional states and sleep quality: a single-center prospective cohort study. J Headache Pain 18:23

23. Blumenfeld AM, Stark RJ, Freeman MC, Orejudos A, Manack AA (2018) Longterm study of the efficacy and safety of OnabotulinumtoxinA for the prevention of chronic migraine: COMPEL study. J Headache Pain 19:13

24. Boudreau GP, Grosberg BM, McAllister PJ, Lipton RB, Buse DC (2015) Prophylactic onabotulinumtoxinA in patients with chronic migraine and comorbid depression: an open-label, multicenter, pilot study of efficacy, safety and effect on headache-related disability, depression, and anxiety. Int J Gen Med 8:79-86

25. Butera C, Colombo B, Bianchi F et al (2016) Refractory chronic migraine: is drug withdrawal necessary before starting a therapy with onabotulinum toxin type a? Neurol Sci 37:1701-1706

26. Demiryurek BE, Ertem DH, Tekin A, Ceylan M, Aras YG, Gungen BD (2016) Effects of onabotulinumtoxinA treatment on efficacy, depression, anxiety, and disability in Turkish patients with chronic migraine. Neurol Sci 37:1779-1784

27. Dominguez C, Vieites-Prado A, Perez-Mato M et al (2018) CGRP and PTX3 as predictors of efficacy of Onabotulinumtoxin type a in chronic migraine: an observational study. Headache 58:78-87

28. Dominguez C, Pozo-Rosich P, Torres-Ferrus M et al (2018) OnabotulinumtoxinA in chronic migraine: predictors of response. A prospective multicentre descriptive study. Eur J Neurol 25:411-416

29. Grazzi L, Usai S (2014) Botulinum toxin a: a new option for treatment of chronic migraine with medication overuse. Neurol Sci 35(Suppl 1):37-39

30. Khalil M, Zafar HW, Quarshie V, Ahmed F (2014) Prospective analysis of the use of OnabotulinumtoxinA (BOTOX) in the treatment of chronic migraine; real-life data in 254 patients from Hull, U.K. J Headache Pain 15:54

31. Kollewe K, Escher CM, Wulff DU et al (2016) Long-term treatment of chronic migraine with OnabotulinumtoxinA: efficacy, quality of life and tolerability in a real-life setting. J Neural Transm 123:533-540

32. Lee MJ, Lee C, Choi H, Chung CS (2016) Factors associated with favorable outcome in botulinum toxin a treatment for chronic migraine: a clinicbased prospective study. J Neurol Sci 363:51-54

33. Naderinabi B, Saberi A, Hashemi $M$ et al (2017) Acupuncture and botulinum toxin a injection in the treatment of chronic migraine: a randomized controlled study. Caspian J Intern Med 8:196-204

34. Negro A, Curto M, Lionetto L, Crialesi D, Martelletti P (2015) OnabotulinumtoxinA $155 \mathrm{U}$ in medication overuse headache: a two years prospective study. Springerplus 4:826

35. Negro A, Curto M, Lionetto L, Martelletti P (2016) A two years open-label prospective study of OnabotulinumtoxinA $195 \mathrm{U}$ in medication overuse headache: a real-world experience. J Headache Pain 17:1

36. Pedraza Ml, de la Cruz C, Ruiz M et al (2015) OnabotulinumtoxinA treatment for chronic migraine: experience in 52 patients treated with the PREEMPT paradigm. Springerplus 4:176

37. Russo M, Manzoni GC, Taga A et al (2016) The use of onabotulinum toxin a (Botox $((R)))$ in the treatment of chronic migraine at the Parma headache Centre: a prospective observational study. Neurol Sci 37:1127-1131

38. Sarchielli P, Romoli M, Corbelli I et al (2017) Stopping Onabotulinum treatment after the first two cycles might not be justified: results of a reallife monocentric prospective study in chronic migraine. Front Neurol 8:655

39. Vikelis M, Argyriou AA, Dermitzakis EV, Spingos KC, Mitsikostas DD (2016) Onabotulinumtoxin-a treatment in Greek patients with chronic migraine. J Headache Pain 17:84

40. Tassorelli C, Sances G, Avenali M, et al (2018) Botulinum toxin for chronic migraine: clinical trials and technical aspects. Toxicon 147:111-115

41. Matharu M, Pascual J, Nilsson Rl et al (2017) Utilization and safety of onabotulinumtoxinA for the prophylactic treatment of chronic migraine from an observational study in Europe. Cephalalgia 37:1384-1397

42. Cady RK, Schreiber CP, Porter JA, Blumenfeld AM, Farmer KU (2011) A multi-center double-blind pilot comparison of onabotulinumtoxinA and topiramate for the prophylactic treatment of chronic migraine. Headache 51:21-32

43. Mathew NT, Jaffri SF (2009) A double-blind comparison of onabotulinumtoxina (BOTOX) and topiramate (TOPAMAX) for the prophylactic treatment of chronic migraine: a pilot study. Headache 49: 1466-1478

44. Frampton JE, Silberstein S (2018) OnabotulinumtoxinA: a review in the prevention of chronic migraine. Drugs 78:589-600 
45. Tassorelli C, Jensen $\mathrm{R}$, Allena $\mathrm{M}$ et al (2017) The added value of an electronic monitoring and alerting system in the management of medication-overuse headache: a controlled multicentre study. Cephalalgia 37:1115-1125

46. Munksgaard SB, Bendtsen L, Jensen RH (2012) Detoxification of medicationoveruse headache by a multidisciplinary treatment programme is highly effective: a comparison of two consecutive treatment methods in an openlabel design. Cephalalgia 32:834-844

47. Westergaard ML, Munksgaard SB, Bendtsen L, Jensen RH (2016) Medicationoveruse headache: a perspective review. Ther Adv Drug Saf 7:147-158

48. Green MW, Rothrock JF (2018) An academic debate: OnabotulinumtoxinA for chronic migraine: PREEMPT-derived vs "customized" dosing/injection paradigm. Toxicon 147:116-119

49. Farrar JT, Young JP Jr, LaMoreaux L, Werth JL, Poole RM (2001) Clinical importance of changes in chronic pain intensity measured on an 11-point numerical pain rating scale. Pain 94:149-158

50. Tassorelli C, Aguggia M, De TM et al (2017) Onabotulinumtoxin a for the management of chronic migraine in current clinical practice: results of a survey of sixty-three Italian headache centers. J Headache Pain 18:66-77

51. Serrano D, Lipton RB, Scher Al et al (2017) Fluctuations in episodic and chronic migraine status over the course of 1 year: implications for diagnosis, treatment and clinical trial design. J Headache Pain 18:101-0787

52. Minen MT, Begasse De DO, Kroon Van DA et al (2016) Migraine and its psychiatric comorbidities. J Neurol Neurosurg Psychiatry 87:741-749

53. Hepp Z, Bloudek LM, Varon SF (2014) Systematic review of migraine prophylaxis adherence and persistence. J Manag Care Pharm 20:22-33

54. Ruggeri M (2014) The cost effectiveness of Botox in Italian patients with chronic migraine. Neurol Sci 35(Suppl 1):45-47

55. Rothrock JF, Bloudek LM, Houle T, Andress-Rothrock D, Varon SF (2014) Real-world economic impact of onabotulinumtoxinA in patients with chronic migraine. Headache 54:1565-1573

56. Batty AJ, Hansen RN, Bloudek LM et al (2013) The cost-effectiveness of onabotulinumtoxinA for the prophylaxis of headache in adults with chronic migraine in the UK. J Med Econ 16:877-887

57. Andreou AP, Trimboli M, Al-Kaisy A et al (2018) Prospective real-world analysis of OnabotulinumtoxinA in chronic migraine post-National Institute for health and care excellence UK technology appraisal. Eur J Neurol 25:1069-1083

Ready to submit your research? Choose BMC and benefit from:

- fast, convenient online submission

- thorough peer review by experienced researchers in your field

- rapid publication on acceptance

- support for research data, including large and complex data types

- gold Open Access which fosters wider collaboration and increased citations

- maximum visibility for your research: over $100 \mathrm{M}$ website views per year

At $\mathrm{BMC}$, research is always in progress.

Learn more biomedcentral.com/submissions 